\title{
COMPARATIVE EXTRACTION OF ESSENTIAL OILS OF Mentha piperita (MINT) BY STEAM DISTILLATION AND ENFLEURAGE
}

\author{
M. Ibrahim*, G. E. Ankwai, J. R. Gungshik and P. Taave \\ ${ }^{1}$ Department of Chemistry, University of Jos, Nigeria. \\ *Corresponding Adress: mibrahim@unijos.edu.ng
}

\begin{abstract}
The extraction of essential oils is generally carried out by two main techniques: azeotropic distillation (hydrodistillation, hydrodiffusion, and steam distillation) and extraction with solvents. This work consists in studying the two methods of extraction of the essential oils of Mentha piperita: Steam distillation (azeotropic) and Enfleurage (solvent extraction). The optimum yield for the extraction of essential oil via steam distillation from Mentha piperita was obtained at 540 minutes with production coming to an end at 1080 minutes yielding $1.36 \%$ of essential oil at 80 ${ }^{\circ} \mathrm{C}$ per $240 \mathrm{~g}$ of sample while extraction through effleurage give maximum yield at 900 minutes with production coming to an end at 1080 minutes. The extraction process recorded a percentage yield of $0.91 \%$ at $80^{\circ} \mathrm{C}$. In view of the above result, it implies that at a given condition the steam distillation method comparatively is a better technique for extraction of essential for optimal production. In addition, the compounds with the highest GC-MS area percentage in the analysis of Mentha piperitta were 1-Decanol, 2-hexyl- (3.8471\%) and L-Menthol which also has a high area percentage (3.5439\%) which is of utmost importance as the fragrance of mint oil extract and its peculiar flavour can be attributed to the abundant presence of levo-menthol. Levo-menthol is used for the treatment, control, prevention, and improvement of the following diseases, conditions and symptoms which includes occasional minor irritation, Pain, Sore mouth, Sore throat and Cough. It can be drawn without doubt, that steam distillation offers significant advantages comparatively over effleurage and can therefore be best suitable for pilot scale extraction of essential oils in Mint.
\end{abstract}

Keywords: Enfleurage, Hydro distillation, Hydro diffusion, and Steam Distillation

\section{INTRODUCTION}

Enfleurage is a process that uses odorless fats that are solid at room temperature to capture the fragrant compounds exuded by plants. However, Steam distillation is an alternative method of achieving distillation at temperatures lower than the normal boiling point. It is applicable when the material to be distilled is immiscible (incapable of mixing) and chemically nonreactive with water. $^{5}$

The most popular Mints for commercial cultivation and use are; peppermint (Mentha piperita), native spearmint (Mentha spicata), 
scotch spearmint (menthe gracilis), and corn mint (Menthe arvensis) also, apple mint (Menthe suaveolens). ${ }^{6}$ Mint are fast growing, extending their reach along surfaces through a network of runners .For this reason, one plant of each desired mint along with a little care will provide more than enough mint for small scale use. All Mints thrive near pools of water, lakes, rivers and cool moist spot in partial shade. Mints tolerate a wide range of conditions and can be grown in full sun and all year round. Mints are planted in deep bottomless containers sunk in the ground and above ground in tubs and barrels which enables their control in opening environment. ${ }^{4}$

Leaves of Mentha have a distinct aroma and cool flavour yielding a pale yellow shade of oil. ${ }^{1}$ It has very high menthol content, also containing vitamin $\mathrm{A}$ and $\mathrm{C}$, potassium, omega 3 fatty acids, iron, magnesium, calcium, and copper. ${ }^{2}$ Mint is characterized by the preponderance of menthone, isomenthone, and different isomers of menthol. Mint thrives near pools of water, lakes, rivers and cool moist spot in partial shade. $^{7}$

Mint is a calming and soothing herb that aids indigestion and stomach upset by increasing bile secretion. It is helpful when dealing with congestion as Compounds Contained in mint help in opening up the nasal passages as well as those of the lungs and bronchi .It is helpful for allergies and asthma due to its antifungal properties. It contains a phytonutrient called perillyl alcohol which has been shown to prevent the formation of skin, colon, and lung cancer. $^{3}$

This research aims at the comparative extraction of essential oils of mentha piperita (mint) by two methods: steam distillation and Enfleurage

\section{MATERIALS AND METHODS}

\section{Sample collection}

Fresh mint sample was collected during early morning hours from a garden at National Institute for Policy and Strategic Studies (NIPSS) Kuru, Plateau state Nigeria.

\section{Sample Preparation}

The Sample of Mint was properly washed and allowed to dry for 30 minutes in the laboratory. The leaves samples were then cut into tiny slices to increase surface for contact with solvent during extraction process. 


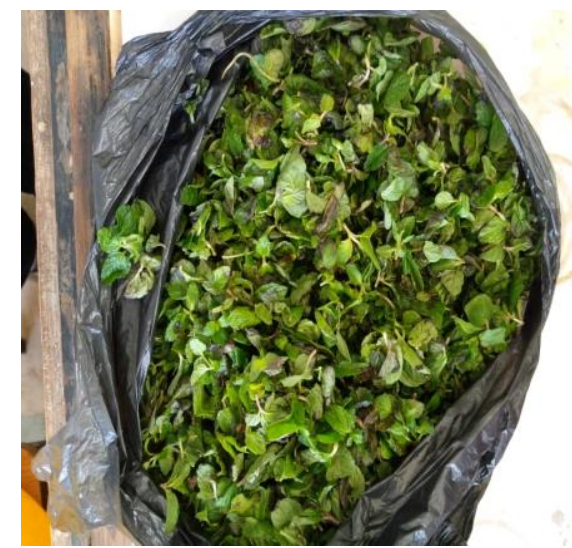

Plates 1: Chopped mint leaves (Mentha
piperitta)

\section{Steam Distillation}

Exactly $60 \mathrm{~g}$ of fresh mint leaves was weighed and placed in a $500 \mathrm{ml}$ round bottom flask containing $250 \mathrm{ml}$ of distilled water and small granulated substances known as antibombing agents was also added to prevent flush when the sample starts boiling. The flask was fitted with a rubber stopper connected to a condenser and gradually heated to a temperature of $80{ }^{\circ} \mathrm{C}$. At $100{ }^{\circ} \mathrm{C}$ it started boiling ripping off the essential oil from the sample substrate. As the heating process continues, essential oil that was extracted from the sample mixed with the water vapour and collected over the condenser by a receiver bottle. Cooling of the condensate was made possible using ice to avoid the volatilization of essential oil.

The condensate was then transferred into a separating funnel forming two distinct layers

Nigerian Journal of Chemical Research of oil and water with the oil being the top layer due to its low density in comparison with the density of water. The tap of the separating funnel was opened to let out the water while the oil was immediately collected into a $100 \mathrm{ml}$ sample bottle. The bottle was closed tightly to prevent vaporization of the essential oil and weighed. This procedure was repeated four times for each sample type respectively

\section{Enfleurage}

Exactly $60 \mathrm{~g}$ of the dry sample were weighed out and pounded with mortar and pestle to increase the surface area reveal. The pounded sample was transferred into a $500 \mathrm{ml}$ beaker and warmed $70 \mathrm{ml}$ of light-flavored olive oil was mixed with the mashed sample (to allow for efficient absorption of the essential oil). The beaker was covered with aluminum foil and shaken until the sample was distributed throughout the oil. It was then allowed to stand for 24 hours at room temperature for proper absorption. Ethanol $140 \mathrm{ml}$ was added to absorb the essential oil leaving behind the light-flavoured olive oil and the sample residue. The Ethanol extract was decanted and placed on a water bath at $80{ }^{\circ} \mathrm{C}$ to vaporize the Ethanol leaving behind the essential oil. The yield of oil was determined by weighing on an electronic weighing 
balance. The difference between the final weight of the beaker and the initial weight gave the yield of essential oil. This procedure was repeated four times for each sample type respectively.

\section{RESULTS AND DISCUSSION}

\section{The oil yields (g) versus Time (mins)}

The results of the variaction in weight of oil extracted from mentha piperita at $80{ }^{\circ} \mathrm{C}$ through steam distillation and effleurage are presented using graph in Fig 1;
The optimum yield for the extraction of essential oil via steam distillation from Mentha piperita was obtained at 540 minutes with production coming to an end at 1080 minutes yielding $1.36 \%$ of essential oil at 80 ${ }^{0} \mathrm{C}$ per $240 \mathrm{~g}$ of sample while extraction through effleurage give maximum yield at 900 minutes with production coming to an end at 1080 minutes. The extraction process recorded a percentage yield of $0.91 \%$ at 80 ${ }^{0} \mathrm{C}$. In view of the above result, it can be inferred that the steam distillation method is a better technique for extraction of essential for optimal production.

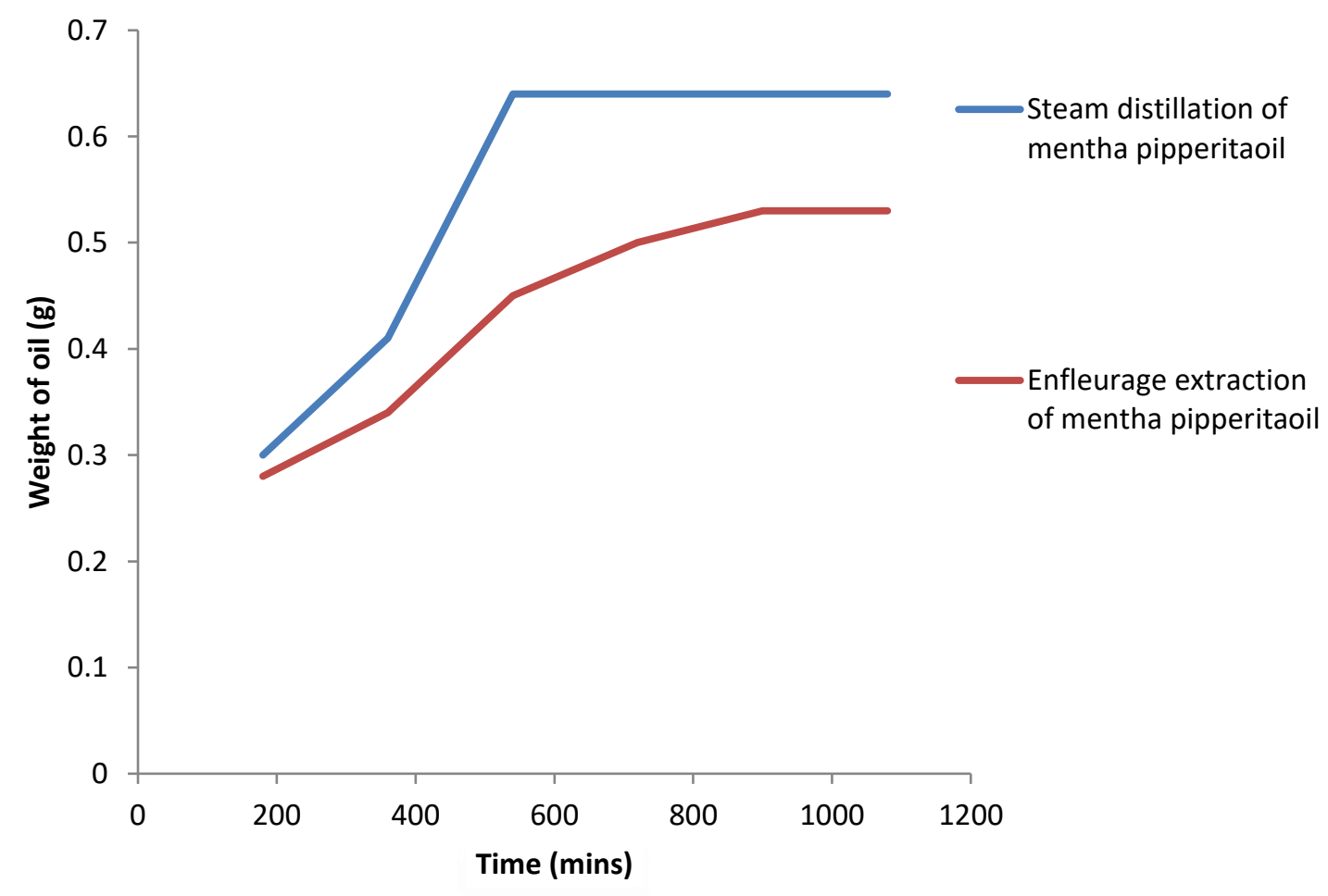


Fig 1: Weight of Mentha peperita Oil Vs Time

Table 1: Percentage Essential oil yield from Mentha piperita

\begin{tabular}{|l|l|}
\hline $\begin{array}{l}\text { Method of } \\
\text { extraction }\end{array}$ & $\begin{array}{l}\text { Percentage(\%) } \\
\text { yield }\end{array}$ \\
\hline $\begin{array}{l}\text { Steam } \\
\text { distillation }\end{array}$ & 1.36 \\
\hline Enfleurage & 0.91 \\
\hline
\end{tabular}

The result in Table 1 above shows that steam distillation is more effective in the extraction of essential oil from mint plants than the enfleurage extraction. This is probably due to the loss of volatile components during the pounding process that preceded extraction.
Identification of constituents was done on the basis of their retention time and mass spectra library search. The mass spectrums of the identified constituents are given in tables 4.3 below. The relative amount of individual components was calculated based on GC peak areas. The GC-MS spectrum obtained revealed that the essential oils of mint contained certain terpenes that eluted at different retention times depending on the boiling point of the eluted component. The GC chromatogram obtained revealed a high concentration of Levomenthol and 1Decanol, 2-hexyl- which is identify by presence of relatively large area percentages of 3.5439 and 3.8471 respectively. 


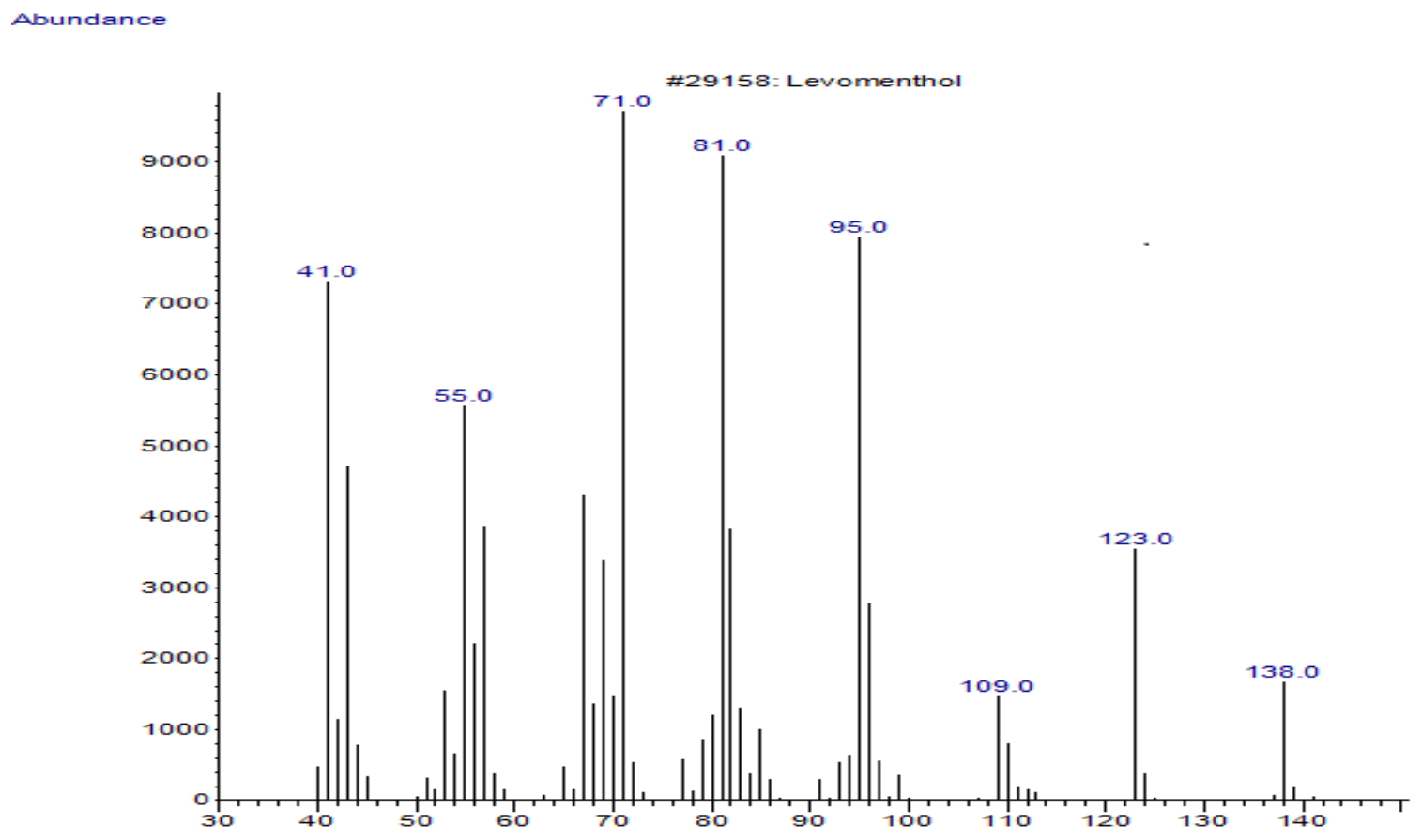

$m v z->$

Fig 2 GC-MS Chromatogram for Mint oil extract

Table 3: Identified Compounds from GC-MS Chromatogram of Mint oil extract

\begin{tabular}{|c|c|c|c|c|c|c|}
\hline s/n & $\begin{array}{c}\text { Library Id (Molucular Formular } \\
\text { And Weight) }\end{array}$ & $\begin{array}{c}\text { Molecular } \\
\text { Formular }\end{array}$ & $\begin{array}{c}\text { Retention } \\
\text { Time }\end{array}$ & $\begin{array}{c}\text { Area } \\
\text { Percentage }\end{array}$ & $\begin{array}{c}\text { Molecular } \\
\text { Class }\end{array}$ & $\begin{array}{c}\text { Molecular } \\
\text { Weight }\end{array}$ \\
\hline 1 & Isobutyl tetracosyl ether & $\mathrm{C}_{28} \mathrm{H}_{58} \mathrm{O}$ & 30.0631 & 1.1332 & Ether & $410.8 \mathrm{~g} / \mathrm{mol}$ \\
\hline 2 & 1 -decosene & $\mathrm{C}_{22} \mathrm{H}_{44}$ & 31.3471 & 2.5845 & $\begin{array}{c}\text { Aliphatic } \\
\text { Alkene }\end{array}$ & $308.6 \mathrm{~g} / \mathrm{mol}$ \\
\hline 3 & Tricosane & $\mathrm{C}_{23} \mathrm{H}_{48}$ & 32.8638 & 2.3195 & Alkane & $324.6 \mathrm{~g} / \mathrm{mol}$ \\
\hline 4 & Eicosane & $\mathrm{C}_{20} \mathrm{H}_{42}$ & 32.3934 & 2.8359 & Alkane & $282.5 \mathrm{~g} / \mathrm{mol}$ \\
\hline 5 & Levomenthol & $\mathrm{C}_{10} \mathrm{H}_{20} \mathrm{O}$ & 11.7843 & 3.5439 & Mono & $156.7 \mathrm{~g} / \mathrm{mol}$ \\
\hline 6 & Hexatricontylpentafluoropropanioate & & 31.148 & 1.2742 & Ester & Alcohol \\
\hline 7 & 1-Decanol, 2-methyl- & $\mathrm{C}_{11} \mathrm{H}_{24} \mathrm{O}$ & 32.565 & 1.0106 & $172 \mathrm{~g} / \mathrm{mol}$ \\
\hline 8 & Estra-1,3,5(10)-trien-17.beta.-ol & $\mathrm{C}_{23} \mathrm{H}_{32} \mathrm{O}_{2}$ & 29.1979 & 1.2399 & Alcohol & $340.5 \mathrm{~g} / \mathrm{mol}$ \\
\hline 9 & Carbonic acid, octadecyl vinyl ester & $\mathrm{C}_{21} \mathrm{H}_{40} \mathrm{O}_{3}$ & 28.4173 & 1.0485 & Acid Ester & $340.5405 \mathrm{~g} / \mathrm{mol}$ \\
\hline 10 & 1-Decanol, 2-hexyl- & $\mathrm{C}_{16} \mathrm{H}_{34} \mathrm{O}$ & 31.2522 & 3.8471 & Alcohol & $242.44 \mathrm{~g} / \mathrm{mol}$ \\
\hline
\end{tabular}


The compound with the highest area percentage in the analysis of Mentha piperitta was 1-Decanol, 2-hexyl$(3.8471 \%)$. Although for the purpose of perfume production, L-Menthol which also has a high area percentage $(3.5439 \%)$ is of utmost importance as the fragrance of mint oil extract and its peculiar flavour can be attributed to the abundant presence of levomenthol. Levo-menthol is used for the treatment, control, prevention, and improvement of the following diseases, conditions and symptoms which includes; occasional minor irritation, Pain, Sore mouth, Sore throat and Cough.

\section{CONCLUSION}

Steam distillation and enfleurage are both suitable techniques that can be used to extract essential oil components from Mint plants. However, the results of the variation of essential oil yields from mint with respect to time at constant temperature shows that extraction through steam distillation gave a better percentage yield for Mentha piperita than the effleurage method. It can therefore be inferred that steam distillation at a conditional temperature can be adopted as a proficient cost effective method for extraction of essential oil compounds from plants for the purpose of perfume making.

The extraction of essential oils by distillation is governed by the sensitivity of the essential oil to the action of heat, water and alcohol (thus essential oils with high solubility in water are susceptible to damage by action of heat therefore cannot be steam distilled). The oil must be steam volatile for steam distillation to be feasible. Most of the essential oils of commercial purposes are steam volatile, reasonably stable to action of heat and practically insoluble in water and hence suitable for processing by steam.

These methods of extraction are special types of separation processes used for heat sensitive materials like essential oils, resins, hydrocarbons, etc. which are insoluble in water and may decompose at their boiling point. The temperature of the steam must be high enough to vaporize the essential oil present, yet not destroy or burns the essential oil compounds. It can therefore be emphasized that Mentha piperita contains essential oil components in substantial amount that can be suitable for perfume formulation and production.

\section{REFERENCE}


1. Ate Tezel; Hortacsu A.; Hortacsu O., (1960). Multi-component Models for Seed and Essential Oil Extraction. Supercritical Fluids, Pp 131-167.

2. Aziz E. E., Abbass M. H., (2010) American-Eurasian of agricultural and environmental science. Vol.8 No.4 pp 411-419.

3. Edris A E (2007) Pharmaceutical and therapeutic potentials of essential oils and their individual volatile constituents: a review. Phytother Res 21: 308-323

4. Harley Raymond M. ,Atkins Sandy, Budantsev Andrey L. ,Cantino Philpstoun D., Conn Barry J., Grayer Renée J., Harley Madelline M., De Kok , Roger P.J., Krestovskaja
Tatyana V (2004). The families and genera of vascular plants view

5. Hesham H. A. Rassem, Abdurahman H. Nour, Rosli M. Yunus, (2016) Techniques For Extraction of Essential Oils From Plants: A Review ISSN 1991-8178

6. Loolaie M, Moasefi N, Rasouli H, Adibi H (2017) Peppermint and Its Functionality: A Review Vol.8 No.4:54

7. Telci Isa, Nermin Sahbaz (2005) Variation of yield, Essential Oil and Carvone Contents in Clones Selected from carvone-scented Landracesof Turkis Metha Species, Journal of agronomy vol. 4 No. 2 pp 96-102. 